\title{
Assessing the performance of monocyte to high-density lipoprotein ratio for predicting ischemic stroke: insights from a population- based Chinese cohort
}

Hao-Yu Wang ${ }^{1,2+}$, Wen-Rui Shi ${ }^{1+}$, Xin $\mathrm{Yi}^{3+}$, Ya-Ping Zhou ${ }^{4}$, Zhi-Qin Wang ${ }^{5}$ and Ying-Xian Sun ${ }^{1 *}$

\begin{abstract}
Background: Monocyte to high-density lipoprotein cholesterol ratio (MHR) is a recently emerged measure of inflammation and oxidative stress and has been used to predict multiple cardiovascular abnormalities, but data relative to ischemic stroke are lacking. The goal of this study was to estimate the associations of MHR and prevalent ischemic stroke among a large cohort of general Chinese population.

Method: The study analyzed 8148 individuals (mean age: 54.1 years; $45.7 \%$ males) enrolled in a cross-sectional population-based Northeast China Rural Cardiovascular Health Study (NCRCHS). We identified 194 patients admitted from January and August 2013 with ischemic stroke.

Results: After adjustment for age, sex, and potential confounders, each standard deviation (SD) increment of MHR was predictive to a greater odd of ischemic stroke (odds ratio, 1.276; 95\% confidence interval [CI], 1.082-1.504), with subjects in the highest quartile of MHR levels having a 1.6-fold higher risk of prevalent ischemic stroke $(95 \% \mathrm{Cl}$, 1.045-2.524) as compared with those in the lowest quartile. Moreover, smoothing curve showed a linear positive pattern of this association. The area under the curve (AUC) significantly increased ( $P=0.042)$ to 0.808 ( $95 \% \mathrm{Cl}$, 0.779-0.837) when the combined MHR was added to the baseline logistic regression model with ischemic stroke risk factors. Also, MHR (0.004) significantly improved integrated discrimination improvement when added to the baseline model.

Conclusions: The present study demonstrated for the first time a linear relation between MHR levels and the odds of ischemic stroke in a large community-based population. The MHR, a marker of high atherosclerotic burden, demonstrated incremental predictive value over traditional clinical risk factors, thus providing clinical utility in risk stratification in subjects presenting with ischemic stroke. These findings had implications for strategies aimed at lowering MHR to prevent adverse cardiovascular and cerebrovascular outcomes.
\end{abstract}

Keywords: Monocyte to high-density lipoprotein ratio, Ischemic stroke, Atherosclerosis, Dyslipidemia, Inflammation, Monocyte, Epidemiology

\footnotetext{
* Correspondence: sunyingxian1962@163.com

${ }^{+}$Hao-Yu Wang, Wen-Rui Shi and Xin Yi contributed equally to this work.

'Department of Cardiology, The First Hospital of China Medical University,

155 Nanjing North Street, Heping District, Shenyang 110001, China

Full list of author information is available at the end of the article
}

(c) The Author(s). 2019 Open Access This article is distributed under the terms of the Creative Commons Attribution 4.0 International License (http://creativecommons.org/licenses/by/4.0/), which permits unrestricted use, distribution, and reproduction in any medium, provided you give appropriate credit to the original author(s) and the source, provide a link to the Creative Commons license, and indicate if changes were made. The Creative Commons Public Domain Dedication waiver (http://creativecommons.org/publicdomain/zero/1.0/) applies to the data made available in this article, unless otherwise stated. 


\section{Introduction}

Over the past decades, stroke has emerged as a major burden of healthcare system [1]. In 2013, stroke affected 1596 per 100,000 people and attributed to 114.8 new deaths per 100,000 people in China [2]. Moreover, the burden of stroke in China appears to be increasing particularly in rural areas, of which ischemic stroke constituted $69.6 \%$ [2, 3]. Indeed, ischemic stroke has become a predominant cause of death and disability in the low income population [4]. There is thus a clear need to identify ischemic stroke at an early stage, and as a result, primary prevention forms the cornerstone of management.

Atherosclerosis, especially intracranial atherosclerosis, is an intrinsic abnormality in the development of ischemic stroke [5-7]. Pathophysiological studies have identified intracranial plaques can cause artery-to-artery emboli, in-situ thromboembolism, hemodynamic impairment and local branch occlusion, which play critical roles in the onset of ischemic stroke $[8,9]$. Additionally, clinical studies revealed the high prevalence of intracranial atherosclerosis among ischemic stroke patients in different races through advanced imaging techniques or autopsy [10-13]. However, advanced imaging techniques are expensive, time-consuming and unavailable in resource-poor settings, and therefore, a novel marker that can be used both clinically and in research studies as intermediate or surrogate outcomes to help early identification and prevention of ischemic stroke is needed.

Inflammation and lipid abnormalities have been proposed as the main constituents of the pathophysiology of atherosclerosis development and progression [14, 15]. Monocytes, hallmarks of chronic inflammation, interact primarily with platelets and endothelial cells resulting in aggravation of inflammatory, pro-thrombotic pathways and are known to play an active role in the formation, progression and rupture of an atherosclerotic plaque at the vascular level $[16,17]$. Conversely, the high-density lipoprotein-cholesterol (HDL-C) protect endothelial cells from inflammation and oxidative stress through controlling monocyte activation and proliferation of monocyte progenitor cells, as well as suppressing the migration of macrophages and oxidation of low-density lipoprotein (LDL) molecules $[18,19]$. In view of the pathogenesis of atherosclerosis, HDL-C level is a lipid parameter that reduces in the presence of endothelial dysfunction and atherosclerosis, and monocyte is a hematological index that increase during inflammation. In this regard, theoretically, HDL-C levels decline while monocyte levels increase in cases of atherosclerosis, thus, the MHR value is expected to increase.

From these observations, a model constructed using these two indices, that is, the emerging monocyte-to-HDL-C ratio (MHR), has been put forward to evaluate the prognosis of cardiovascular events by assessing atherosclerosis [20]. Later studies also revealed its utility in the prediction of hallmarks of cardiovascular outcomes, such as metabolic syndrome, coronary artery disease, and atrial fibrillation recurrence [21-25]. However, no study to date has evaluate the association between MHR and ischemic stroke. Therefore, this study aimed to investigate the impact of MHR on ischemic stroke and explore the value of MHR to stratify the risk of ischemic stroke based on 8148 participants of the general population from China.

\section{Materials and methods}

\section{Study population}

The present study originated from a large population-based cross-sectional epidemiological investigation named Northeast China Rural Cardiovascular Health Study (NCRCHS). After excluding people with pregnancy, malignant tumor and mental disease, the survey recruited a total of 11,956 participants (age $\geq 35$ years) between January 2013 and August 2013 at 26 rural villages across Liaoning province in China. Details regarding the design and rationale of NCRCHS has been fully displayed elsewhere [26-28]. In the present study, 3808 participants were further excluded due to their missing values of covariates. Finally, 8148 subjects (mean age: 54.1 years, male: $45.7 \%$ ) were carried into statistical analyses (Fig. 1). Our study protocol was approved by the Ethic Committee of China Medical University (Shenyang, China) and the whole data and procedures conformed to the principle of ethical standards. Every enrolled participant provided a written informed consent.

\section{Data collection and measurements}

Prior studies have delineated data collection and measurements in detail [26-28]. In brief, after cardiologists and nursed completed a training course and passed an exam, they were certificated for conducting questionnaires which collected data about demographic information, health-related behavior, anthropometric parameters and the use of lipidlowering drug (at least one type versus no). Subjects were asked to indicate their average use of food items during the previous year for assessment of the dietary patterns. We used a food frequency questionnaire to collect baseline information on dietary habits in terms of the average consumption of several food items per week. The reported consumption was quantified approximately according to the grams per week. Vegetable consumption was rated on the following scale: scored 0, 1, 2, and 3 for $\geq 2000 \mathrm{~g}, 1000-2000 \mathrm{~g},<1000 \mathrm{~g}$, and rare level, respectively. Meat consumption, including red meat, fish and poultry, was classified into 4 categories as follows: rarely $=0 \mathrm{~g} /$ wk., $<250 \mathrm{~g} / \mathrm{wk} .=1,250-500 \mathrm{~g} / \mathrm{wk} .=2$ and $\geq 500$ $\mathrm{g} / \mathrm{wk} .=3$. A diet score was generated by adding the vegetable consumption score (from 0 to 3 ) plus meat consumption score (from 0 to 3 ) on a scale of $0-6$ for each participant, with higher scores indicating greater Westernized diet adherence. Meanwhile, a central steering committee with a subcommittee was employed to fulfill the quality control process of these collected data. 


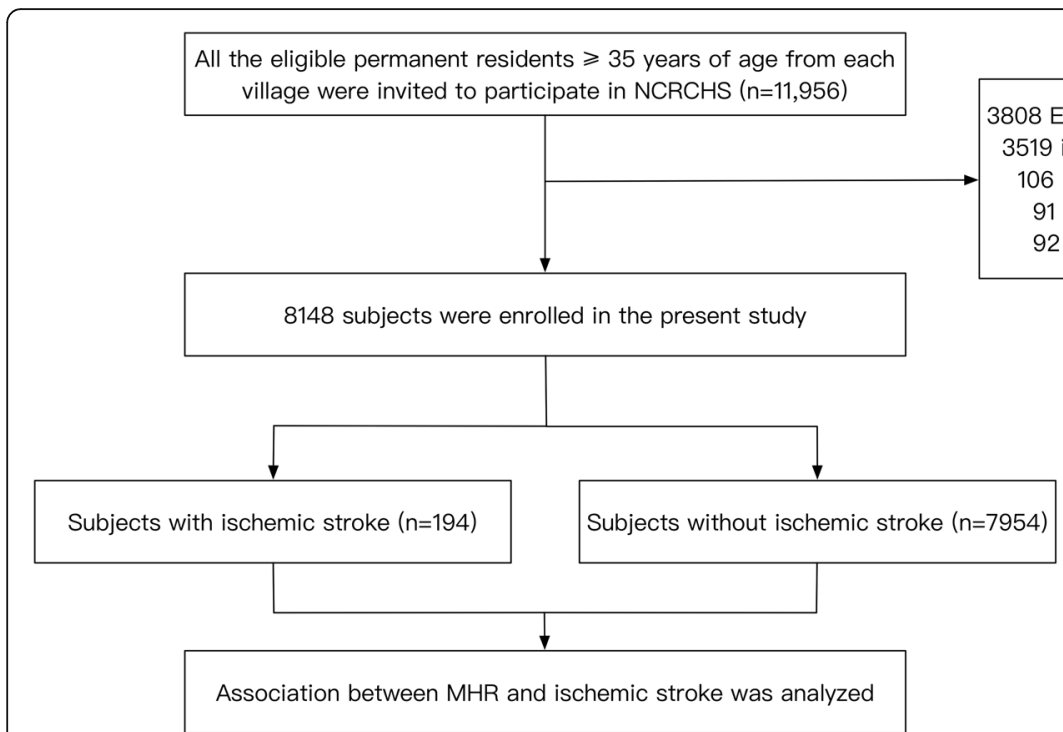

Fig. 1 Flowchart describing the selection process and derivation of the study population

Study participants waited for at least $5 \mathrm{~min}$ in a relaxed and sitting position. Then blood pressure (BP) was measured by a standardized automatic electronic sphygmomanometer (HEM-907; Omron, Kyoto, Japan) using an appropriately sized cuff with the arm supported at the level of the heart. The mean readings of three replicate measurements were recorded for the present analysis.

Anthropometric indexes were collected when subjects only wore light clothing without shoes. The weight of subjects was quantified to the nearest $0.1 \mathrm{~kg}$ by a calibrated digital scale. After subjects held in a standing position, their height was recorded by a portable stadiometer with a scale of $0.1 \mathrm{~cm}$. An elastic measuring tape was utilized to obtain the waist circumference (WC) horizontally at $1 \mathrm{~cm}$ above the umbilicus. All of the above measurements were conducted twice and their mean values were included into the analyses. The subjects were reminded of keeping an overnight fasting with $12 \mathrm{~h}$ before the investigation. Details about the process of blood sample transportation, storage and laboratory tests were extensively described in our previous studies [26-28].

\section{Definitions}

Body mass index (BMI) was calculated as mean weight divided by mean height squared $\left(\mathrm{kg} / \mathrm{m}^{2}\right)$. MHR was defined as blood monocyte count ratio to high-density lipoprotein cholesterol concentration [20]. Hypertension was recognized as systolic blood pressure (SBP) $\geq 140 \mathrm{mmHg}$ and / or diastolic blood pressure (DBP) $\geq 90 \mathrm{mmHg}$, subjects with self-reported current antihypertensive medication or previous diagnosed hypertension were also considered as hypertensive patients [29]. Diagnosis of diabetes based on fasting plasma glucose (FPG) $\geq 7.0 \mathrm{mmol} / \mathrm{L}$ and/or selfreported previous diagnosed diabetes, subjects receiving plasma glucose lowering therapy at baseline were also regarded as diabetic patients [30].

Based on strict neurological examination, computed tomography or magnetic resonance imaging, first-ever ischemic stroke was defined as stroke event with a diagnosis of thrombosis or embolism. Documentation of ischemic stroke reviewed by two independent neurologists was confirmed on the basis of the information on diagnostic tests or hospital records.

\section{Statistical analyses}

Continuous variables were expressed as mean values \pm standard deviation (SD) or median (interquartile range) according to their distributions. Category variables were presented as frequencies (percentages). Before inferential analyses, MHR values were log transformed due to highly skewed distributions. Comparisons of continuous variables were conducted by Student's t test or Mann-Whitney test. Chi-square test was occupied to compare categorical variables between groups. Additionally, to compare ordinal categorical variables (education level, family annual income, physical activity) between groups, rank-sum test was employed to utilize the ordinal information. Adjusted odds ratios (ORs) and 95\% confidence intervals (CIs) for the independent association between MHR and the prevalent ischemic stroke were determined using a multivariate logistic regression model adjusted for potential confounding covariates. The confounding variables used for adjustment included age, sex, race, education, family annual income, physical activity status, current smoking and drinking conditions, diet score, serum creatinine $(\mathrm{Scr})$, SBP, FPG, BMI, and lipid-lowering drug. Analyses were conducted with MHR as continuous variables (per SD increase) and then categorized as quartiles. The results were displayed as odds ratios (ORs) and $95 \%$ confidence intervals $(95 \% \mathrm{CI})$. 
A generalized additive model (GAM) with a spline smoothing function was applied to examine the relationship between MHR and the risk of ischemic stroke. Subgroup analysis examined the relationship between MHR and the risk of ischemic stroke according to age, sex, SBP $(<140$ and $\geq 140$ $\mathrm{mmHg})$, FPG $(<7.0$ and $\geq 7.0 \mathrm{mmol} / \mathrm{L})$, and $\mathrm{BMI}(<28$ and $\geq$ $28 \mathrm{~kg} / \mathrm{m}^{2}$ ). Test for interaction in the logistic-regression model was used to compare odd ratios (ORs) between the analyzed subgroups. The incremental predictive value of MHR in addition to clinical risk factors, was assessed using the Harrell's $\mathrm{C}$ index, net reclassification improvement (NRI), and the integrated discrimination improvement index (IDI). Clinical risk factors included age, sex, current smoking, current drinking, Scr, SBP, FPG and BMI. All of the statistical analyses were performed by SPSS 25.0 software (IBP corp), statistical software packages R (http://www.r-project.org, The R Foundation) and EmpowerStats (http://www.empowerstats.com, X\&Y Solutions, Inc., Boston, MA). A two-tailed $P$ value less than 0.05 was recognized as significant.

\section{Results}

Table 1 summarizes the data of 8148 eligible subjects (males: 45.7\%). The prevalence of ischemic stroke was $2.38 \%$. As for the demographic data, participants with ischemic stroke were older and had lower education, family annual income and physical activity status than their healthy counterparts. Additionally, ischemic stroke subjects were less likely to be a current smoker or drinker. With regard to the anthropometric characteristics, ischemic stroke group had a significant lower level of height, with the exception of weight and BMI, which were unaltered. Concordantly, adults with ischemic stroke exhibited higher levels of SBP and DBP together with increased SCr and FPG. As expected, participants with ischemic stroke constituted a higher percentage of hypertension, diabetes, and lipid-lowering drug relative to the non-ischemic stroke ones. Regarding lipid profiles, higher total cholesterol, triglyceride and LDL-C were remarkably augmented in ischemic stroke patients compared with their counterparts, while HDL-C concentrations were greatly reduced in ischemic stroke subjects (all $P<0.05$ ). Within our expectation, patients with ischemic stroke experienced pronounced increment of white blood cell count, neutrophil and monocyte counts. Lastly, MHR levels were statistically greater in ischemic stroke subjects than non-ischemic stroke group.

Multivariate logistic regression model demonstrated the association between MHR and ischemic stroke (Table 2). After adjusting for age, sex, race, education, family annual income and physical activity status, current smoking and drinking conditions, and diet score, each SD increment of MHR could cast additional 37.5\% risk of ischemic stroke on subjects. Additional adjustment of Scr, SBP, FPG, BMI, and lipid-lowering drug mildly modified this association, but the full model still had a OR of 1.276 (95\% CI: 1.082, 1.504). After dividing MHR into quartiles, we observed a $62.4 \%$ increase of the risk for ischemic stroke when comparing top quartile with bottom category in the fully adjusted model. Furthermore, the risk of ischemic stroke showed an increasing trend across the quartiles ( $\mathrm{P}$ for trend = 0.024). Additionally, in order to explore the dose-response relationship between MHR and the risk of ischemic stroke, we employed smooth curve fitting with full adjustment of all covariates (Fig. 2). The resultant curve displayed a linear correlation between normalized MHR and ischemic stroke risk. This result was consistent with the increasing trend across the quartiles in the logistic model.

To confirm the findings in logistic models were robust to potential confounders, we conducted stratified analysis by subgroups defined by covariates that had been demonstrated to have major roles in affecting stroke risk, including age, sex, SBP, FPG, and BMI (Fig. 3). All of these analyses were adjusted for age, sex, race, education, family income and physical activity level, current smoking and drinking status, diet score, Scr, SBP, FPG, BMI, and lipid-lowering drug except for the covariate that was stratified. Figure 3 revealed a highly consistent pattern: the risk for ischemic stroke increased with greater MHR regardless of subgroups (all $\mathrm{P}$ for interaction $>0.05$ ).

The areas under the receiver operator characteristic curves of the multivariable logistic regression models are shown in Table 3. For ischemic stroke, the baseline logistic regression model with clinical risk factors (age, sex, current smoking, current drinking, Scr, SBP, FPG and BMI) had a C-statistic of 0.802 (95\% CI, 0.773$0.831)$. Adding MHR significantly increased $(P=0.042)$ the C-statistic to $0.808(95 \% \mathrm{CI}, 0.779-0.837)$. The IDI but not the category free NDI was significantly improved when MHR was added to the baseline model adjusted for clinical risk factors.

\section{Discussion}

Our study examined for the first time the relationship between the MHR and prevalence of ischemic stroke in a large community-based population. The principal findings of our study are two-fold. First, elevated MHR levels were dose dependently associated with increasing odds of ischemic stroke, even after adjusting for traditional ischemic stroke risk factors and other important covariables. MHR may serve as a clinically useful and potentially modifiable inflammation-based marker for identifying patients who are at higher risk for cardiovascular and cerebrovascular outcomes. Second, the MHR has the potential to further refine predictive risk estimation as compared to the classic clinical risk factors, suggesting a method to optimize the prevention of ischemic stroke. Taken together, targeted preventive 
Table 1 Characteristics of subjects stratified by ischemic stroke

\begin{tabular}{|c|c|c|c|}
\hline Variables & Ischemic stroke $(N=194)$ & Non-ischemic stroke $(N=7954)$ & $P$ value $^{a}$ \\
\hline Age (years) & $64.48 \pm 8.87$ & $53.87 \pm 10.39$ & $<0.001$ \\
\hline Male (\%) & $86(44.3)$ & $3641(45.8)$ & 0.690 \\
\hline Race (Han) (\%) & $188(96.9)$ & $7740(97.3)$ & 0.773 \\
\hline Education level (\%) & & & $<0.001$ \\
\hline Primary school or below & $142(73.2)$ & $4171(52.4)$ & \\
\hline Middle school & $40(20.6)$ & 3091 (38.9) & \\
\hline High school or above & $12(6.2)$ & $692(8.7)$ & \\
\hline Income (CNY) (\%) & & & $<0.001$ \\
\hline$\leq 5000$ & $56(28.9)$ & $795(10.0)$ & \\
\hline $5000-20,000$ & $100(51.5)$ & $4183(52.6)$ & \\
\hline$>20,000$ & $38(19.6)$ & $2976(37.4)$ & \\
\hline Physical activity (\%) & & & $<0.001$ \\
\hline Low & $142(73.2)$ & $3038(38.2)$ & \\
\hline Middle & $24(12.4)$ & $1522(19.1)$ & \\
\hline High & $28(14.4)$ & $3394(42.7)$ & \\
\hline Current smoking (\%) & $53(27.3)$ & $2712(34.1)$ & 0.049 \\
\hline Current drinking (\%) & $21(10.8)$ & $1712(21.5)$ & $<0.001$ \\
\hline Diet score & $1.72 \pm 1.15$ & $2.28 \pm 1.14$ & $<0.001$ \\
\hline Height (cm) & $158.46 \pm 8.12$ & $160.72 \pm 8.14$ & $<0.001$ \\
\hline Weight (kg) & $62.53 \pm 9.54$ & $63.53 \pm 11.31$ & 0.153 \\
\hline $\mathrm{BMI}\left(\mathrm{kg} / \mathrm{m}^{2}\right)$ & $24.91 \pm 3.41$ & $24.53 \pm 3.61$ & 0.151 \\
\hline $\mathrm{SBP}(\mathrm{mmHg})$ & $154.16 \pm 25.31$ & $138.29 \pm 21.71$ & $<0.001$ \\
\hline $\mathrm{DBP}(\mathrm{mmHg})$ & $85.85 \pm 11.88$ & $81.76 \pm 11.60$ & $<0.001$ \\
\hline $\operatorname{Scr}(\mu \mathrm{mol} / \mathrm{L})$ & 78.60 (70.48-88.63) & $73.70(66.60-82.10)$ & $<0.001$ \\
\hline FPG (mmol/L) & $5.84(5.38-6.59)$ & $5.60(5.22-6.09)$ & $<0.001$ \\
\hline $\mathrm{TC}(\mathrm{mmol} / \mathrm{L})$ & $5.48 \pm 1.06$ & $5.31 \pm 1.11$ & 0.038 \\
\hline $\mathrm{TG}(\mathrm{mmol} / \mathrm{L})$ & $1.81(1.17-2.69)$ & $1.29(0.91-1.95)$ & $<0.001$ \\
\hline $\mathrm{HDL}-\mathrm{C}(\mathrm{mmol} / \mathrm{L})$ & $1.24 \pm 0.28$ & $1.34 \pm 0.32$ & $<0.001$ \\
\hline LDL-C (mmol/L) & $3.01 \pm 0.80$ & $2.89 \pm 0.80$ & 0.033 \\
\hline WBC count $\left(10^{9} / \mathrm{L}\right)$ & $6.30(5.30-7.60)$ & $6.00(4.90-7.10)$ & $<0.001$ \\
\hline Neutrophil count $\left(10^{9} / \mathrm{L}\right)$ & $3.80(3.00-4.64)$ & $3.40(2.70-4.30)$ & $<0.001$ \\
\hline Lymphocyte count $\left(10^{9} / \mathrm{L}\right)$ & $1.90(1.60-2.40)$ & $1.90(1.60-2.40)$ & 0.593 \\
\hline Monocyte count $\left(10^{9} / \mathrm{L}\right)$ & $0.50(0.35-0.68)$ & $0.41(0.30-0.60)$ & 0.004 \\
\hline Hypertension (\%) & $167(86.1)$ & $3642(45.8)$ & $<0.001$ \\
\hline Diabetes (\%) & $46(23.7)$ & $828(10.4)$ & $<0.001$ \\
\hline Lipid-lowering drug (\%) & $30(15.5 \%)$ & $242(3.0 \%)$ & $<0.001$ \\
\hline MHR & $0.39(0.27-0.55)$ & $0.34(0.23-0.48)$ & $<0.001$ \\
\hline
\end{tabular}

Data are expressed as mean \pm standard deviation (SD) or median (interquartile range) and numbers (percentage) as appropriate

Abbreviations: $C N Y$ Chinese currency ( $1 C N Y=0.15$ USD), BMI body mass index, SBP systolic blood pressure, DBP diastolic blood pressure, Scr serum creatinine, FPG fasting plasma glucose, TC total cholesterol; TG triglyceride, HDL-C high-density lipoprotein cholesterol, LDL-C low-density lipoprotein cholesterol, WBC white blood cell, MHR monocyte count to high-density lipoprotein ratio

${ }^{a}$ Comparisons of category variables between groups were tested by chi-square test or rank-sum test (ordinal category variables) and comparisons for continuous variables between groups were tested by Student's t or Mann-Whitney test 
Table 2 Evaluation of the impact of MHR on ischemic stroke by multivariate logistic regression models

\begin{tabular}{|c|c|c|c|c|c|c|}
\hline \multirow[t]{2}{*}{ Variables } & \multicolumn{6}{|l|}{ Odds Ratio $(95 \%$ Cl) } \\
\hline & Crude & $P$ value & Model 1 & $P$ value & Model 2 & $P$ value \\
\hline MHR (Per 1 SD increase) & $1.426(1.221,1.666)$ & $<0.001$ & $1.375(1.170,1.615)$ & $<0.001$ & $1.276(1.082,1.504)$ & 0.004 \\
\hline \multicolumn{7}{|l|}{ Quartiles of MHR } \\
\hline Quartile 1 & 1.000 (reference) & & 1.000 (reference) & & 1.000 (reference) & \\
\hline Quartile 2 & $1.314(0.831,2.076)$ & 0.243 & $1.206(0.756,1.925)$ & 0.432 & $1.155(0.720,1.851)$ & 0.550 \\
\hline Quartile 3 & $1.434(0.915,2.248)$ & 0.116 & $1.393(0.880,2.207)$ & 0.157 & $1.257(0.788,2.005)$ & 0.338 \\
\hline Quartile 4 & $2.186(1.440,3.319)$ & $<0.001$ & $1.924(1.252,2.956)$ & 0.003 & $1.624(1.045,2.524)$ & 0.031 \\
\hline$P$ for trend & & $<0.001$ & & 0.001 & & 0.024 \\
\hline
\end{tabular}

Abbreviations: MHR monocyte count to high-density lipoprotein cholesterol ratio, OR odds ratio; 95\% Cl: 95\% confidence interval, SD standard deviation. Other abbreviations as in Table 1

Crude: no adjustment; Model 1: adjusted for age, sex, race, education level, family annual income level, physical activity, current smoking, current drinking, and diet score; Model 2: adjusted for all the factors in model 1 and serum creatinine, systolic blood pressure, fasting plasma glucose, body mass index, and lipid-lowering drug

Quartile 1: MHR $<0.229$; Quartile 2: $0.229 \leq M H R<0.341$; Quartile 3: $0.341 \leq M H R<0.485$; Quartile 4: MHR $\geq 0.485$

programs that reduce the burden of inflammation and lipid abnormalities as estimated by MHR, possibly will reduce the prevalent ischemic stroke at both levels of primary and secondary prevention.

Accelerated atherosclerosis in intracranial arteries is an intrinsic nature of ischemic stroke [5-7]. Artery-artery embolism, hypoperfusion, and branch atheromatous disease are the likely mechanisms for ischemic stroke in intracranial atherosclerosis $[8,9]$. Furthermore, clinical studies also employed advanced imaging techniques and autopsy to evaluate the direct correlation between intracranial atherosclerosis and ischemic stroke, and they identified the high prevalence of intracranial atherosclerosis among ischemic stroke patients in different races [10-13]. More importantly, the prevalence of intracranial atherosclerosis and mortality of ischemic stroke were extremely high, especially in Asian population $[6,10,12,31]$. Although intracranial atherosclerosis is a good marker of general illness related to chronic diseases states that may predispose to the development of ischemic stroke, the techniques to detect intracranial atherosclerosis at now are expensive, time-consuming and unavailable in resource-poor settings. Thus, a simple, stable and cost-effective index of atherosclerosis to refine the risk stratification of ischemic stroke may be needed.

Inflammation and lipid abnormalities are two interplayed hallmarks of atherosclerosis, driving the healing response to vascular injury and allowing the initiation and growth of atherosclerotic plaque $[14,15,32]$. Monocytes act as an important source of pro-inflammatory species during atherosclerosis process $[16,17,33]$. This

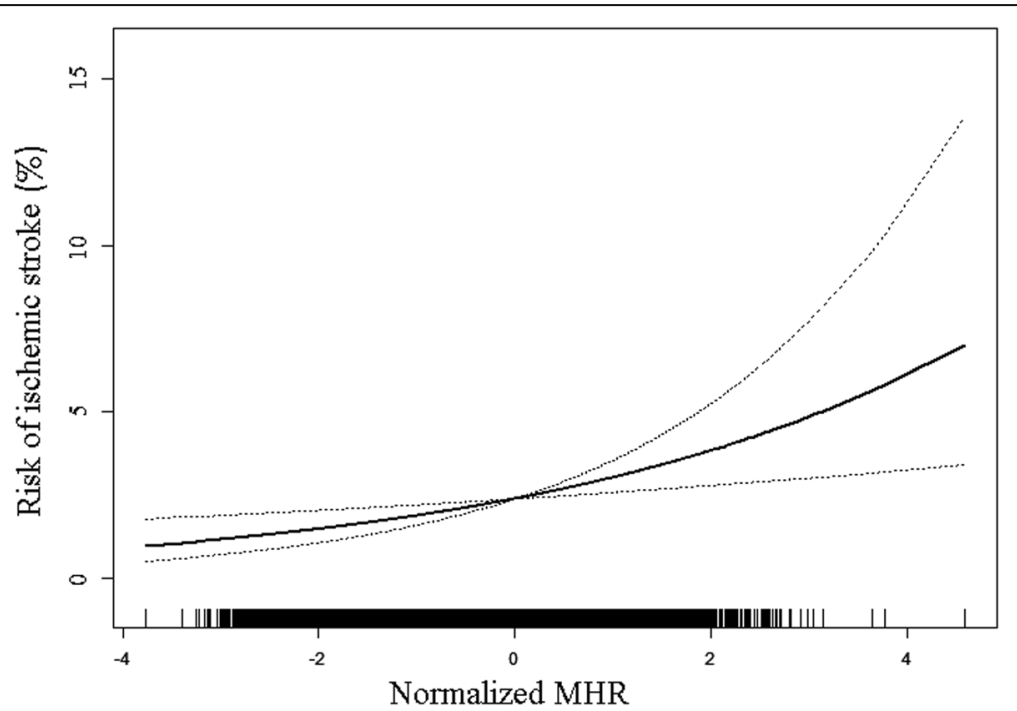

Fig. 2 Smooth curve fitting was performed using generalized additive model to explore the association between MHR and the risk of ischemic stroke after adjusting for age, sex, race, education level, family annual income level, physical activity, current smoking, current drinking, diet score, serum creatinine, systolic blood pressure, fasting plasma glucose, body mass index, and lipid-lowering drug. In this figure, the solid line indicates the estimated risk of ischemic stroke while the dotted lines serve as pointwise $95 \%$ confidence intervals 


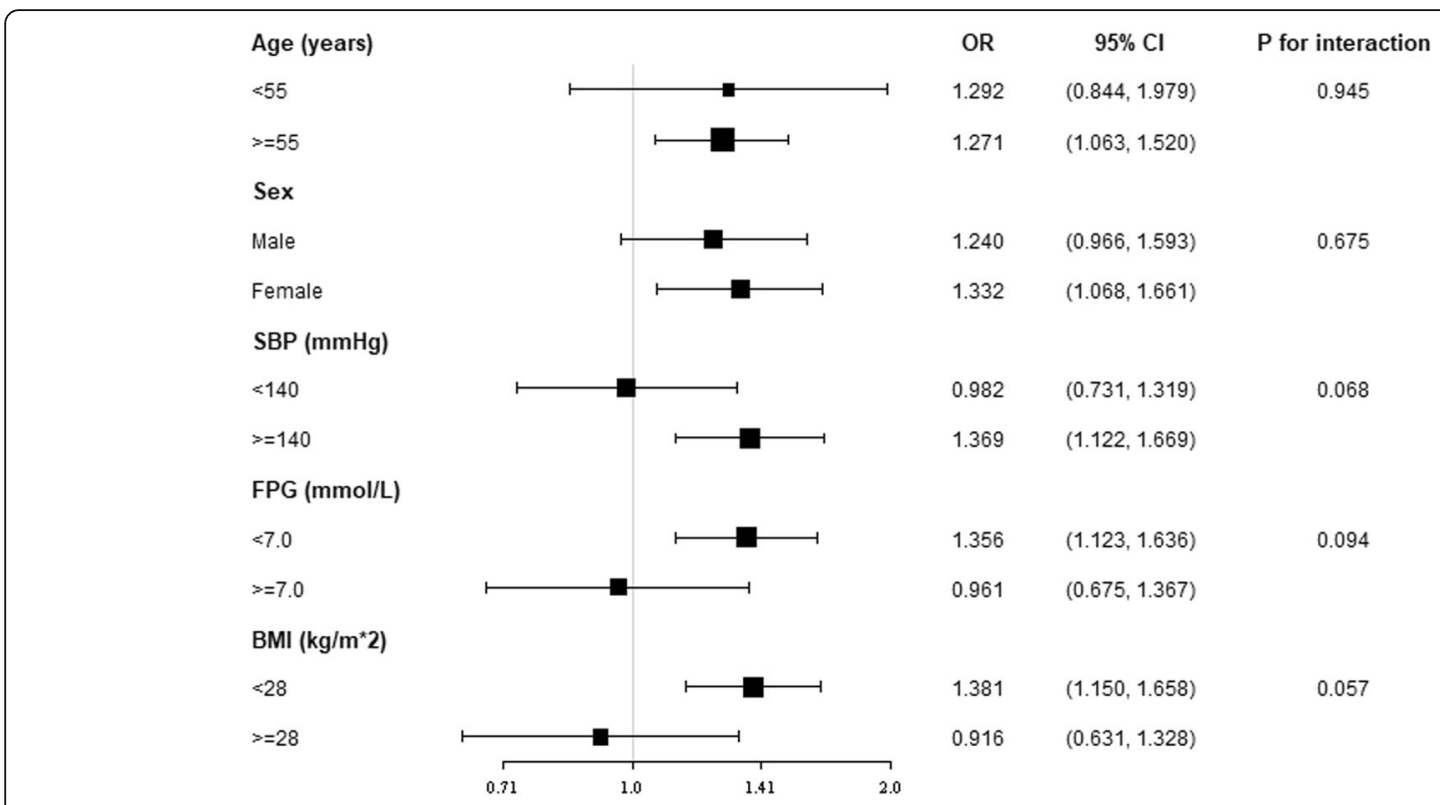

Fig. 3 Subgroup analyses on impact of MHR on the prevalence of ischemic stroke. The dots and lines indicate the estimates of the odds ratios of ischemic stroke for each SD increment of MHR and the corresponding 95\% confidence intervals, respectively. The model adjusted for age, sex, race, education level, family annual income level, physical activity, current smoking, current drinking, diet score, serum creatinine, systolic blood pressure, fasting plasma glucose, body mass index, and lipid-lowering drug, except for the variable that is stratified

suggests that activated monocytes interact with damaged or activated endothelium, characterized by the overexpression of proinflammatory cytokines/adhesion molecules. Then, monocytes infiltrate into vascular subendothelial space and mature into macrophages [14, 15]. These macrophages further engulf oxidized LDL-C and transform into foam cells. Then the foam cells release inflammatory cytokines which recruit more monocytes into the pathological foci. Therefore, inflammatory cholesterol ester-loaded plaque is formed [34]. Hence, it has been shown that high monocyte counts are important determinant of atherosclerotic diseases, especially among persons with coronary artery disease and ischemic stroke [35-37]. On the other end of the spectrum, HDL-C facilitates the reverse cholesterol transportation from peripheral vessels to liver, reducing the lipid accumulation in peripheral [38, 39]. Moreover, HDL-C neutralizes the pro-inflammatory and pro-oxidant effects of monocytes by inhibiting LDL-C oxidation in vascular wall and preventing monocyte recruitment into vascular wall $[18,19]$, thereby suppressing the proliferation of monocyte progenitor cells and controlling monocyte activation [40]. As expected, these steps protect endothelial cells from inflammation and oxidative stress. Based on these mechanisms, the relationship between reduced HDL-C levels and atherosclerotic diseases, such as coronary heart disease and ischemic stroke is well established in some prospective cohorts [41, 42]. Notably, monocytes are relevant to proinflammatory and pro-oxidant effect, but HDL-C functions as a reversal factor during atherosclerosis process. On the assumption that monocytes and HDL-C have apparent functions in the progression or inhibition of atherosclerosis, therefore, incorporation of monocytes and HDL-C into MHR create an improved index that included HDL-C that is found at low levels in the presence of atherosclerosis, as well as monocyte frequency, which is known to increase in this setting. Also, the results of the CANTOS study provided strong support for the hypothesis that inflammation is a treatable pathogenic mechanism in atherosclerosis [32].

MHR is a newly proposed index to estimate atherosclerosis by assessing inflammation and dyslipidemia [20]. Studies have identified the utility of MHR to predict multiple atherosclerotic diseases, such as intra-stent restenosis, coronary artery disease, infective endocarditis and metabolic syndrome, with the emphasis placed on MHR as a novel risk marker of

Table 3 Comparison of the risk stratifying ability of MHR in addition to clinical risk factors

\begin{tabular}{|c|c|c|c|c|c|c|}
\hline Model & C-Statistic (95\% Cl) & $P$ value & NRI (category free) & $P$ value & $\mathrm{IDI}$ & $P$ value \\
\hline Clinical risk factors $^{a}$ & $0.802(0.773,0.831)$ & Reference & Reference & Reference & Reference & Reference \\
\hline Clinical risk factors + MHR & $0.808(0.779,0.837)$ & 0.042 & $0.128(-0.013,0.269)$ & 0.075 & $0.004(0.000,0.007)$ & 0.0043 \\
\hline
\end{tabular}

C-Statistic, net reclassification improvement (NRI) and integrated discrimination improvement (IDI) were compared between models

${ }^{a}$ The reference model included risk factors only, including age, sex, current smoking, current drinking, serum creatinine, systolic blood pressure, fasting plasma glucose, and body mass index 
rapidly assessing systemic inflammatory response and possible endothelial dysfunction [23-25, 43]. It is probable that an increased MHR was suggestive for inflammation that exerts its primary actions in atherosclerosis development and progression and, hence, ischemic stroke. In fact, a growing body of evidence indicates the role of inflammation in the pathogenesis of ischemic stroke [44-46]. Experimentally and clinically, acute and prolonged inflammatory process have been observed in cerebral ischemic injury, that is, the rapid activation of resident cells (mainly microglia), production of proinflammatory mediators, and infiltration of various types of inflammatory cells (including neutrophils, $\mathrm{T}$ cells, monocyte/macrophages, and other cells) into the ischemic brain tissue [47]. During the acute phase of ischemic stroke, injured tissue releases proinflammatory mediators (cytokines and chemokines) and reactive oxygen species, eventually promoting the adhesion and migration of circulating leukocytes $[48,49]$. In the subacute phase, leukocytes infiltration potentiates the local inflammatory response, characterized by increased production of cytokines and chemokines, and activation of matrix metalloproteinase, which in turn result in junctional proteins cleavage and disruption of blood brain barrier integrity, thus feeding the deleterious inflammatory loop $[48,50]$. On the basis of these findings and pathophysiological role of inflammation in ischemic stroke, we hypothesized that higher MHR would be associated with an increased odd of ischemic stroke and can be utilized as an ideal marker to improve the risk stratification of ischemic stroke. Furthermore, it is known that the large artery atherosclerosis and cardioembolic causes have been acknowledged as major determinants for ischemic stroke. As mentioned earlier, high MHR levels have been observed in patients with vascular risk factors associated with atherosclerosis and cardiovascular disease [21-25, 51]. Thus, MHR has a documented role in these processes or may modify the risk of ischemic stroke by modulating the conventional risk factor pathway. Importantly, atrial fibrillation (AF) is the most frequent reason for cardioembolic stroke. In a prospective and observational study with 402 cases of symptomatic AF, elevated MHR was correlated with an increased recurrence of after cryoballoon-based catheter ablation [21]. Taken together, the effects of MHR levels on ischemic stroke may be mediated through components of the inflammation and pathways of atrial fibrillation. Hence, it might be speculated that high MHR ratio may represent an additional independent risk factor for ischemic stroke.

Our observation of a positive association between MHR and the odds of having ischemic stroke was line with the hypothesis. These findings were independent of key sociodemographic and potentially modifiable vascular risk factors in a rural community-based sample. Higher MHR levels were associated with significantly greater odds of ischemic stroke in multivariable adjusted models. In a recent prospective study among 397 individuals with current smoking and 515 age-matched healthy participants without history of smoking, higher MHR levels have been found to be associated with cigarette smoking [51]. It is particularly notable that the strong association between elevated MHR and prevalent ischemic stroke was independent of current smoking status in our data. Additional studies are warranted to confirm the influence of amount of daily smoking and duration of smoking (pack.year) on this association. Furthermore, the smooth curve fitting validated this association was linear in the whole range of MHR value. Therefore, when using MHR as an indicator of the risk of ischemic stroke, a higher MHR value means a higher risk of ischemic stroke, and there is no threshold or saturation effect existing in this association. This discovery affirmed the stability of MHR to stratify the risk of ischemic stroke. Additionally, the association persisted in the stratification analysis of sex, age, SBP, FPG and BMI, suggesting the risk stratifying ability of MHR is applicable to a wide range of subjects.

In the ROC analysis, we observed a significant improvement for the stroke identifying ability after adding MHR into a model of clinical risk factors (including sex, age, current smoking and drinking status, Scr, SBP, FPG, and BMI). This finding evidenced the usefulness of MHR to improve risk stratification of ischemic stroke. For more accurate determination of the risk stratifying ability of MHR, we performed reclassification analysis by calculating category free NRI and IDI. The result was intriguing. Although IDI confirmed a significant risk reclassification by introducing MHR, the category free NRI remained statistically insignificant. The reason of this discrepancy can be explained by the low statistical efficiency of category free NRI $[52,53]$. Algorism of category free NRI can only recognize 2 types of change in risk reclassification: upward or downward. The algorism cannot utilize the degree of the change, therefore a large upward or downward change in risk classification will cause the same category free NRI result as a small upward or downward change respectively [53], thus the category free NRI may not recognize the improvement from MHR. However, with a full usage of reclassification data, IDI successfully found out this advancement [52]. Overall, we could conclude that MHR brought a significant improvement to the risk stratification of ischemic stroke.

There are possible mechanistic explanations for the relation between MHR and ischemic stroke. First, reduced HDL-C level results in impaired reverse transportation of cholesterol from peripheral vessels to liver, leading to accumulation of lipids in arteries [54, 55]. The accumulated lipids undergo peroxidation and form oxidized phospholipids, then the products experience further hydrolyzation and derive into lysophosphatidylcholine (LPC), which mediates the major atherogenic activity of oxidized lipids [56]. As a result, elevated LPC level promotes endothelial cells to express adhesion molecules and chemo-attractants [57, 58]. Furthermore, the dysregulated lipid profiles also promote the production of circulating 
monocytes from hematopoietic stem cells [59, 60]. In addition, HDL-C reduction leads to decreased inhibition of the proinflammatory and pro-oxidant function of monocytes $[18,19]$. Consequently, the over-loaded pro-inflammatory monocytes are recruited to the pathologic foci by endothelial cells released substances and then maturate into macrophages [61, 62]. These macrophages convert into foam cells through internalization of accumulated lipids. The foam cells further transform into a key component of atherosclerotic core through necrosis [15, 63]. Finally, the progressively aggravated intracranial atherosclerosis plays a critical role in the formation of artery to artery embolism, hemodynamic hypoperfusion, local branch occlusion and in-situ thrombotic occlusion, which are fundamental mechanism of ischemic stroke [64].

There are several study limitations. Due to the crosssectional nature of our study, and no causal conclusions can be made about the temporal relationship of MHR with prevalent ischemic stroke. Second, the study is representative of the general population in the rural area of northeast China that limits the generalizability of our findings to other ethnicities with different economic condition. Third, although multivariable adjustments were performed for potential confounding factors, a possible effect of various inflammatory biomarkers such as C-reactive protein, interleukin-6, and oxidative stress biomarkers cannot be excluded. Lastly, as in any observational epidemiologic study, residual confounding by uncollected or unknown risk factors can introduce bias into our results. Yet, results were robust to adjustments for multiple major risk factors. Strengths of our study include a large population-based design, well-documented and standardized data collection instruments, and a relatively large number of ischemic stroke events to power the overall study.

\section{Conclusions}

In summary, we were able to show for the first time that MHR was an independent predictor of ischemic stroke among rural Chinese adults and demonstrated incremental predictive value over traditional clinical risk factors, thus providing clinical utility in risk stratification in subjects presenting with ischemic stroke. Our findings also contributed to the understanding of a potential role of systemic inflammation-related mechanisms in the formation of ischemic stroke and encourage further investigation of therapeutic strategies aimed at fostering a reduction in inflammation and lipid accumulation, as reflected by MHR levels.

\section{Abbreviations}

AF: Atrial fibrillation; BMI: Body mass index; Cl: Confidence interval; CNY: Chinese yuan, 1CNY = 0.15USD; DBP: Diastolic blood pressure; FPG: Fasting plasma glucose; HDL-C: High-density lipoprotein cholesterol; IDI: Integrated discrimination improvement; LDL-C: Low-density lipoprotein cholesterol; LPC: Lysophosphatidycholine; MHR: Monocyte count to highdensity lipoprotein cholesterol ratio; NCRCHS: Northeast China Rural Cardiovascular Health Study; NRI: Net reclassification improvement; OR: Odds ratio; ROC: Receiver operating characteristic curve; SBP: Systolic blood pressure; Scr: Serum creatinine; SD: Standard deviation; WC: Waist circumference

\section{Acknowledgements}

We would like to express our gratitude to all those who exert their effects in achieving this study.

\section{Authors' contributions}

In this study, HYW, WRS, and XY did the study design, statistical analyses and results interpretation. YPZ and ZQW participated as analyzing and resolving difficulties of analytic strategies and results discussion. Finally, YXS functioned as final reviewer and corresponding author. All authors read and approved the final manuscript.

\section{Funding}

This study was supported by grants from "Thirteenth Five-Year" program funds (The National Key Research and Development Program of China, Grant \#2017YFC1307600).

\section{Availability of data and materials}

The datasets used and/or analyzed during the current study are available from the corresponding author on reasonable request.

\section{Ethics approval and consent to participate}

This study was conducted in compliance with the ethical principle of the Declaration of Helsinki. All participants provided written informed consent and all procedures were performed in accordance with the ethical standards. The study protocol was approved by the Ethics Committee of China Medical University (Shenyang, China).

\section{Consent for publication}

All co-authors and participants have given their consent for publication of this article in Lipids in Health and Disease.

\section{Competing interests}

The authors declare that they have no competing interests.

\section{Author details}

${ }^{1}$ Department of Cardiology, The First Hospital of China Medical University, 155 Nanjing North Street, Heping District, Shenyang 110001, China.

${ }^{2}$ Department of Cardiology, State Key Laboratory of Cardiovascular Disease, Fuwai Hospital, National Center for Cardiovascular Diseases, Chinese Academy of Medical Sciences and Peking Union Medical College, Beijing, China. ${ }^{3}$ Department of Cardiovascular Medicine, Beijing Huimin Hospital, Beijing 100054, China. ${ }^{4}$ Department of Neurology, The First Hospital of China Medical University, 155 Nanjing North Street, Heping District, Shenyang 110001, China. ${ }^{5}$ School of Clinical Medicine, China Medical University, Shenyang 110122, China.

Received: 12 February 2019 Accepted: 22 May 2019

Published online: 30 May 2019

\section{References}

1. Liu L, Wang D, Wong KS, Wang Y. Stroke and stroke care in China: huge burden, significant workload, and a national priority. Stroke. 2011; 42(12):3651-4.

2. Wang $W$, Jiang $B$, Sun $H$, Ru X, Sun D, Wang $L$, et al. Prevalence, incidence, and mortality of stroke in China: results from a Nationwide populationbased survey of 480687 adults. Circulation. 2017:135(8):759-71.

3. Wang J, An Z, Li B, Yang L, Tu J, Gu H, et al. Increasing stroke incidence and prevalence of risk factors in a low-income Chinese population. Neurology. 2015;84(4):374-81.

4. Yang G, Wang Y, Zeng Y, Gao GF, Liang X, Zhou M, et al. Rapid health transition in China, 1990-2010: findings from the global burden of disease study 2010. Lancet. 2013;381(9882):1987-2015.

5. Banerjee C, Chimowitz MI. Stroke caused by atherosclerosis of the major intracranial arteries. Circ Res. 2017;120(3):502-13.

6. Qureshi Al, Caplan LR. Intracranial atherosclerosis. Lancet. 2014; 383(9921):984-98

7. Battistella V, Elkind M. Intracranial atherosclerotic disease. Eur J Neurol. 2014; 21(7):956-62. 
8. Kim JS, Nah HW, Park SM, Kim SK, Cho KH, Lee J, et al. Risk factors and stroke mechanisms in atherosclerotic stroke: intracranial compared with extracranial and anterior compared with posterior circulation disease. Stroke. 2012:43(12):3313-8.

9. Chen H, Hong H, Liu D, Xu G, Wang Y, Zeng J, et al. Lesion patterns and mechanism of cerebral infarction caused by severe atherosclerotic intracranial internal carotid artery stenosis. J Neurol Sci. 2011;307(1-2):79-85.

10. Wong K, Li H, Chan Y, Ahuja A, Lam W, Wong A, et al. Use of transcranial Doppler ultrasound to predict outcome in patients with intracranial largeartery occlusive disease. Stroke. 2000;31(11):2641-7.

11. White $H$, Boden-Albala B, Wang C, Elkind MS, Rundek T, Wright CB, et al. Ischemic stroke subtype incidence among whites, blacks, and Hispanics: the northern Manhattan study. Circulation. 2005;111(10):1327-31.

12. De Silva DA, Woon FP, Lee MP, Chen CP, Chang HM, Wong MC. South Asian patients with ischemic stroke: intracranial large arteries are the predominant site of disease. Stroke. 2007;38(9):2592-4.

13. Mazighi M, Labreuche J, Gongora-Rivera F, Duyckaerts C, Hauw JJ, Amarenco P. Autopsy prevalence of intracranial atherosclerosis in patients with fatal stroke. Stroke. 2008;39(4):1142-7.

14. Schaftenaar F, Frodermann V, Kuiper J, Lutgens E. Atherosclerosis. Curr Opin Lipidol. 2016:27(3):209-15.

15. Libby P. Inflammation in atherosclerosis. Arterioscler Thromb Vasc Biol. 2012; 32(9):2045-51.

16. Mestas J, Ley K. Monocyte-endothelial cell interactions in the development of atherosclerosis. Trends in cardiovascular medicine. 2008;18(6):228-32.

17. Woollard K, Geissmann F. Monocytes in atherosclerosis: subsets and functions. Nat Rev Cardiol. 2010;7(2):77-86.

18. Parthasarathy S, Barnett J, Fong LG. High-density lipoprotein inhibits the oxidative modification of low-density lipoprotein. Biochim Biophys Acta. 1990;1044(2):275-83.

19. Murphy AJ, Woollard KJ, Hoang A, Mukhamedova N, Stirzaker RA, McCormick SP, et al. High-density lipoprotein reduces the human monocyte inflammatory response. Arterioscler Thromb Vasc Biol. 2008;28(11):2071-7.

20. Kanbay M, Solak Y, Unal H, Kurt Y, Gok M, Cetinkaya H, et al. Monocyte count/HDL cholesterol ratio and cardiovascular events in patients with chronic kidney disease. Int Urol Nephrol. 2014;46(8):1619-25.

21. Canpolat U, Aytemir K, Yorgun H, Şahiner L, Kaya E, Çay S, et al. The role of preprocedural monocyte-to-high-density lipoprotein ratio in prediction of atrial fibrillation recurrence after cryoballoon-based catheter ablation. Europace. 2015;17(12):1807-15.

22. Kundi H, Gok M, Kiziltunc E, Cetin M, Cicekcioglu H, Cetin ZG, et al. Relation between monocyte to high-density lipoprotein cholesterol ratio with presence and severity of isolated coronary artery ectasia. Am J Cardiol. 2015;116(11):1685-9.

23. Ya G, Qiu Z, Tianrong P. Relation of monocyte/high-density lipoprotein cholesterol ratio with coronary artery disease in type 2 diabetes mellitus. Clin Lab. 2018;64(6):901-6.

24. Vahit D, Mehmet K, Samet $Y$, Hüseyin E. Assessment of monocyte to high density lipoprotein cholesterol ratio and lymphocyte-to-monocyte ratio in patients with metabolic syndrome. Biomark Med. 2017:11(7):535-40.

25. Wei XB, Chen F, Huang JL, He PC, Wei YX, Tan N, et al. Novel risk biomarker for infective endocarditis patients with Normal left ventricular ejection fraction- monocyte to high-density lipoprotein cholesterol ratio. Circulation journal : official journal of the Japanese Circulation Society. 2017;82(1):283-8.

26. Wang H, Chen Y, Sun G, Jia P, Qian H, Sun Y. Validity of cardiometabolic index, lipid accumulation product, and body adiposity index in predicting the risk of hypertension in Chinese population. Postgrad Med. 2018;130(3):325-33.

27. Wang H, Wang S, Yi X, Tao Y, Qian H, Jia P, et al. Estimate of ischemic stroke prevalence according to a novel 4-tiered classification of left ventricular hypertrophy: insights from the general Chinese population. Ann Med. 2018;50(6):519-28.

28. Wang H, Sun Y, Li Z, Guo X, Chen S, Ye N, et al. Gender-specific contribution of cardiometabolic index and lipid accumulation product to left ventricular geometry change in general population of rural China. BMC Cardiovasc Disord. 2018;18(1):62.

29. Chobanian A, Bakris G, Black H, Cushman W, Green L, Izzo J, et al. The seventh report of the joint National Committee on prevention, detection, evaluation, and treatment of high blood pressure: the JNC 7 report. JAMA. 2003;289(19):2560-72.

30. American Diabetes Association. 2. Classification and diagnosis of diabetes. Diabetes Care. 2018;41(Suppl 1):S13-27.

31. Johnston SC, Mendis S, Mathers CD. Global variation in stroke burden and mortality: estimates from monitoring, surveillance, and modelling. The Lancet Neurology. 2009;8(4):345-54.
32. Ridker PM, Everett BM, Thuren T, MacFadyen JG, Chang WH, Ballantyne C, et al. Antiinflammatory therapy with Canakinumab for atherosclerotic disease. N Engl J Med. 2017;377(12):1119-31.

33. Swirski FK, Nahrendorf M. Leukocyte behavior in atherosclerosis, myocardial infarction, and heart failure. Science. 2013;339(6116):161-6.

34. Simionescu M. Implications of early structural-functional changes in the endothelium for vascular disease. Arterioscler Thromb Vasc Biol. 2007;27(2):266-74.

35. Choi S, Kim J, Lim S, Lim J, Kim K, Park K, et al. Monocyte count as a predictor of cardiovascular mortality in older Korean people. Age Ageing. 2017;46(3):433-8.

36. Madjid M, Awan I, Willerson JT, Casscells SW. Leukocyte count and coronary heart disease. J Am Coll Cardiol. 2004;44(10):1945-56.

37. Grosse GM, Schulz-Schaeffer WJ, Teebken OE, Schuppner R, Dirks M Worthmann $\mathrm{H}$, et al. Monocyte subsets and related chemokines in carotid artery stenosis and ischemic stroke. Int J Mol Sci. 2016:17(4):433.

38. Rosenson RS, Brewer HB Jr, Ansell BJ, Barter P, Chapman MJ, Heinecke JW, et al. Dysfunctional HDL and atherosclerotic cardiovascular disease. Nat Rev Cardiol. 2016;13(1):48-60

39. Maiolino G, Rossitto G, Caielli P, Bisogni V, Rossi GP, Calo LA. The role of oxidized low-density lipoproteins in atherosclerosis: the myths and the facts. Mediat Inflamm. 2013:2013:714653.

40. Yvan-Charvet L, Pagler T, Gautier EL, Avagyan S, Siry RL, Han S, et al. ATPbinding cassette transporters and HDL suppress hematopoietic stem cell proliferation. Science. 2010;328(5986):1689-93.

41. Di Angelantonio E, Sarwar N, Perry P, Kaptoge S, Ray K, Thompson A, et al. Major lipids, apolipoproteins, and risk of vascular disease. JAMA. 2009; 302(18):1993-2000.

42. Reina S, Llabre M, Allison M, Wilkins J, Mendez A, Arnan M, et al. HDL cholesterol and stroke risk: the multi-ethnic study of atherosclerosis. Atherosclerosis. 2015:243(1):314-9.

43. Yilmaz S, Akboga M, Sen F, Balcı K, Aras D, Temizhan A, et al. Usefulness of the monocyte-to-high-density lipoprotein cholesterol ratio to predict bare metal stent restenosis. Biomark Med. 2016;10(9):959-66.

44. Jin R, Yang G, Li G. Inflammatory mechanisms in ischemic stroke: role of inflammatory cells. J Leukoc Biol. 2010;87(5):779-89.

45. Bonaventura A, Liberale L, Vecchie A, Casula M, Carbone F, Dallegri F, et al. Update on inflammatory biomarkers and treatments in ischemic stroke. Int $J$ Mol Sci. 2016;17(12).

46. McColl BW, Allan SM, Rothwell NJ. Systemic infection, inflammation and acute ischemic stroke. Neuroscience. 2009;158(3):1049-61.

47. Jin R, Liu L, Zhang S, Nanda A, Li G. Role of inflammation and its mediators in acute ischemic stroke. J Cardiovasc Transl Res. 2013;6(5):834-51.

48. Amantea D, Nappi G, Bernardi G, Bagetta G, Corasaniti MT. Post-ischemic brain damage: pathophysiology and role of inflammatory mediators. FEBS J. 2009:276(1):13-26.

49. Yilmaz G, Granger DN. Cell adhesion molecules and ischemic stroke. Neurol Res. 2008:30(8):783-93.

50. Lakhan SE, Kirchgessner A, Tepper D, Leonard A. Matrix metalloproteinases and blood-brain barrier disruption in acute ischemic stroke. Front Neurol. 2013:4:32.

51. Yilmaz M, Kayancicek $\mathrm{H}$. A new inflammatory marker: elevated monocyte to HDL cholesterol ratio associated with smoking. J Clin Med. 2018;7(4).

52. Pencina MJ, D' Agostino RB, D' Agostino RB, Vasan RS. Evaluating the added predictive ability of a new marker: from area under the ROC curve to reclassification and beyond. Stat Med. 2008;27(2):157-72.

53. Pencina M, D'Agostino R, Steyerberg E. Extensions of net reclassification improvement calculations to measure usefulness of new biomarkers. Stat Med. 2011;30(1):11-21.

54. Brufau G, Groen AK, Kuipers F. Reverse cholesterol transport revisited: contribution of biliary versus intestinal cholesterol excretion. Arterioscler Thromb Vasc Biol. 2011;31(8):1726-33.

55. Rader DJ, Alexander ET, Weibel GL, Billheimer J, Rothblat GH. The role of reverse cholesterol transport in animals and humans and relationship to atherosclerosis. J Lipid Res. 2009;50 Suppl:S189-94.

56. Aiyar N, Disa J, Ao Z, Ju H, Nerurkar S, Willette RN, et al. Lysophosphatidylcholine induces inflammatory activation of human coronary artery smooth muscle cells. Mol Cell Biochem. 2007;295(1-2):113-20.

57. Matsumoto T, Kobayashi T, Kamata K. Role of lysophosphatidylcholine (LPC) in atherosclerosis. Curr Med Chem. 2007;14(30):3209-20.

58. Rong J, Berman J, Taubman M, Fisher E. Lysophosphatidylcholine stimulates monocyte chemoattractant protein-1 gene expression in rat aortic smooth muscle cells. Arterioscler Thromb Vasc Biol. 2002;22(10):1617-23. 
59. Tolani S, Pagler TA, Murphy AJ, Bochem AE, Abramowicz S, Welch C, et al. Hypercholesterolemia and reduced HDL-C promote hematopoietic stem cell proliferation and monocytosis: studies in mice and FH children. Atherosclerosis. 2013;229(1):79-85.

60. Seijkens T, Hoeksema MA, Beckers L, Smeets E, Meiler S, Levels J, et al. Hypercholesterolemia-induced priming of hematopoietic stem and progenitor cells aggravates atherosclerosis. FASEB J. 2014;28(5):2202-13.

61. Quinn M, Parthasarathy S, Fong L, Steinberg D. Oxidatively modified low density lipoproteins: a potential role in recruitment and retention of monocyte/macrophages during atherogenesis. Proc Natl Acad Sci U S A. 1987:84(9):2995-8.

62. Rajavashisth T, Andalibi A, Territo M, Berliner J, Navab M, Fogelman A, et al. Induction of endothelial cell expression of granulocyte and macrophage colony-stimulating factors by modified low-density lipoproteins. Nature. 1990;344(6263):254-7.

63. Ross R. Atherosclerosis-an inflammatory disease. N Engl J Med. 1999;340(2):115-26.

64. Wong KS, Caplan LR, Kim JS. Stroke Mechanisms. Front Neurol Neurosci. 2016;40:58-71.

\section{Publisher's Note}

Springer Nature remains neutral with regard to jurisdictional claims in published maps and institutional affiliations.

- fast, convenient online submission

- thorough peer review by experienced researchers in your field

- rapid publication on acceptance

- support for research data, including large and complex data types

- gold Open Access which fosters wider collaboration and increased citations

- maximum visibility for your research: over $100 \mathrm{M}$ website views per year

At $\mathrm{BMC}$, research is always in progress. 